\title{
All-Around 3D Reconstruction from Spherical Images with Semantic Segmentation
}

\author{
Tuulia Pennanen, Siva Ariram, Antti Tikanmäki, and Juha Röning \\ Biomimetics and Intelligent Systems Group \\ University of Oulu, Finland \\ tuulia.pennanen@oulu.fi
}

\begin{abstract}
Lightweight, affordable spherical cameras can be utilized in mobile robotics to build a 3D map of the working environment of a robot. This paper demonstrates how to use the optical flow between two spherical images to quickly construct a semantically meaningful 3D model spanning all directions. The main contribution of the paper is the use of semantic segmentation to increase the robustness of the reconstruction method in both indoor and outdoor applications.
\end{abstract}

Index Terms-3D reconstruction, semantic segmentation, spherical images, mobile robotics

\section{INTRODUCTION}

The development of omnidirectional sensors in recent years has provided new opportunities for the navigation of autonomous mobile robots. 3D Lidar devices are capable of producing a highly accurate 3D map in all directions, but their usability is limited by their price and inability to scan the environment in real time. A more affordable alternative is a spherical (360-degree) camera consisting of two fisheye lenses. The advantages of such a sensor include its affordability, small size, and low weight.

Spherical cameras do not directly provide the 3D map of the environment, but the dense 3D structure can be reconstructed from at least two spherical images captured at different locations. When aiming for the most lightweight and affordable possible solution, a promising approach is to use a single spherical camera moved by a robotic arm or a wheeled robot, for example.

The goal of our research is to develop software that provides a semantically meaningful map from a pair of spherical images and works as close to real time as possible, and thus the accuracy of the $3 \mathrm{D}$ coordinates is given less emphasis than the processing time. Our work is mainly connected to the dense reconstruction method presented in [1], which describes how to use the optical flow between two spherical images to estimate the motion between the two camera poses and how to utilize this information to build a 3D model with an arbitrary metric scale. The follow-up work in [2] discusses the motion estimation when dealing with more than two images. Another dense mapping method based on the optical flow for spherical images is introduced in [3], where a series of images is used to differentiate the reliable optical flow vectors from the unreliable ones. All of these previous approaches are mainly suitable for indoor uses, while our work pays special attention to situations encountered in outdoor environments.

As an alternative to the optical flow approach, the dense mapping techniques in [4] and [5] are applications of offline structure-from-motion approaches modified for spherical cameras. Compared to these methods, the technique presented in [6] is more likely to work in real time through the application of a SLAM (simultaneous localization and mapping) method designed for regular perspective images.

A difference between our approach and the methods mentioned above is that our work relies on semantic information. Semantic classification of spherical images has already been utilized in some localization and mapping applications, such as the 2D indoor localization method in [7], which is based on the identification of sparsely distributed objects. Another idea relying on lower-level semantic categories is presented in [8], where the geometric context of indoor structures is inferred from location and color data. This facilitates the reconstruction of surfaces with a uniform texture, which is also one of the focus areas of our research.

Learning-based approaches for reconstruction have been gaining popularity in recent years, and an application for spherical images is introduced in [9], where a depth map for an indoor scene is computed from a single spherical image. One severe difficulty with the learning approach is the lack of spherical data with ground truth depth information, especially for outdoor scenes.

Compared to all of these methods, the most distinct feature of our approach is using semantic information to increase the robustness of the motion evaluation, particularly in outdoor environments. The main difference compared to [1] is that the camera motion is evaluated solely based on the regions where we except to obtain a reliable estimation of the optical flow. The decision whether to include a region in the motion evaluation is based on its semantic classification. The neural network that performs the semantic segmentation thus has two simultaneous purposes: improving the quality of the reconstruction and constructing an environmental model with both geometric and semantic information. 


\section{3D Reconstruction USING THE Optical Flow BETWEEN SPHERICAL IMAGES}

The $3 \mathrm{D}$ reconstruction method described in this paper is based on the approach explained in [1]. The technique relies on the fact that spherical images contain data from all directions, which can be exploited to estimate the camera motion between two such images. The motion is parameterized by the Euler angles $\alpha, \beta$, and $\gamma$ for rotation, and the latitude and longitude $\theta$ and $\phi$ on the unit sphere corresponding to the epipolar direction, i.e., the direction of the translational motion of the camera. To find the metric scale of the translational movement, it is necessary to utilize an external motion sensor or to observe an object of a known size.

The process of finding the five independent motion parameters begins with an evaluation of the optical flow between the images. Equirectangular projections are used for this purpose, which requires that the optical flow can be estimated in the presence of strong distortions. An example of the optical flow patterns between two images is depicted in Fig. 1. There is little rotation between the camera poses, and thus the motion patterns correspond to translational motion: the flow vectors appear to emerge from the epipole and converge towards its antipode. This behavior could be disrupted in the presence of dynamic objects, uniform textures, or objects at very short or long distances from the camera.

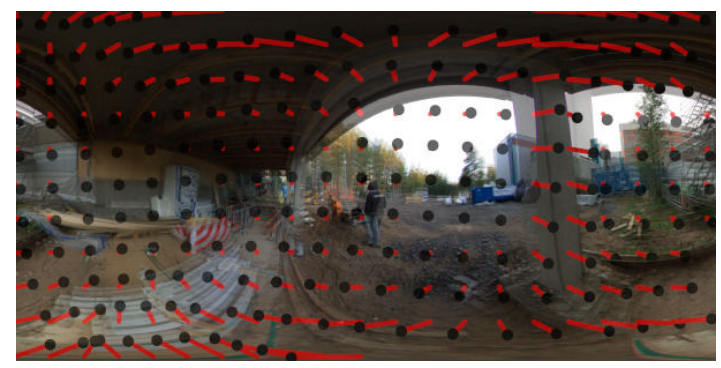

Fig. 1. A nearly ideal example of a purely translational optical flow pattern between two equirectangular images.

From the optical flow vectors, the camera motion parameters are estimated via nonlinear optimization. In each iteration, the optical flow vectors are recalculated to undo the effect of the rotation between the two camera poses. The corrected vectors should result from translational motion alone. In the ideal case, the translation vectors are aligned with the great circles going through the epipoles when transferred to the unit sphere. Considering the flow at each pixel $i$, the direction of a rotation-corrected flow vector $\vec{f}_{i}(\alpha, \beta, \gamma)$ at a point $\vec{x}_{i}$ should be the same as the direction of a vector with the same origin as $\vec{f}_{i}(\alpha, \beta, \gamma)$ but directed tangentially to the epipolar circle. This is equal to observing a zero angle between the plane going through $\vec{x}_{i}$ and $\vec{x}_{i}+\vec{f}_{i}(\alpha, \beta, \gamma)$ and the plane going through $\vec{x}_{i}$ and $\vec{q}(\theta, \phi)$, where $\vec{q}(\theta, \phi)$ is the epipolar direction. Because of this, the motion parameters can be obtained by solving the minimization problem

$$
\underset{\alpha, \beta, \gamma, \theta, \phi}{\operatorname{argmin}} \sum_{\forall i}\left(w_{i} \frac{\left[\vec{x}_{i} \times\left(\vec{x}_{i}+\vec{f}_{i}\right)\right] \cdot\left[\vec{x}_{i} \times \vec{q}\right]}{\left\|\vec{x}_{i} \times\left(\vec{x}_{i}+\overrightarrow{f_{i}}\right)\right\|\left\|\vec{x}_{i} \times \vec{q}\right\|}\right)^{2}
$$

using a weighting function

$$
w_{i}=\sin \theta_{i},
$$

where $\theta_{i}$ is the latitude corresponding to pixel $i$. The function is used to decrease the weight of the distorted pixels at the top and bottom of an equirectangular image. The general idea behind the minimization is illustrated in Fig. 2 .

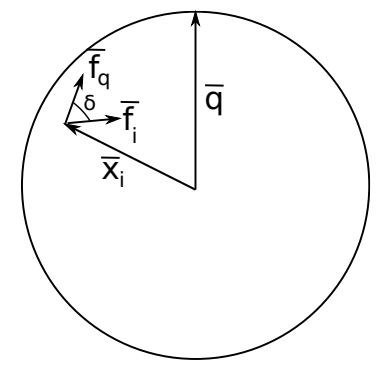

Fig. 2. The motion parameters are found by minimizing the angle $\delta$ between the vectors $\vec{f}_{i}$ and $\overrightarrow{f_{q}}$, where $\overrightarrow{f_{i}}$ is the rotation-corrected optical flow evaluated at pixel $i$ and $\vec{f}_{q}$ is the ideal optical flow vector aligned with the epipolar line going through the epipole and the pixel coordinate on the unit sphere. The unit vectors $\vec{q}$ and $\vec{x}_{i}$ respectively give the locations of the epipole and the 3D position of pixel $i$ on the unit sphere.

The Levenberg-Marquardt algorithm [10] is suitable for such nonlinear minimization problems, and it is also applied in this work. The algorithm requires an initial guess for the set of parameters, which turns out to be a crucial step in the reconstruction task. Due to the presence of local minima, a poor initial guess may lead to convergence far away from the correct values.

Because the reconstruction is based on the translational motion of the camera and the rotational motion is basically just a source of errors in this context, the motion of the camera should be roughly controlled to allow for as little rotation as possible. This task is fairly easy because the camera is required to move a fairly short distance between the frames. The rotation angles are thus initially set to zero: $\alpha=\beta=\gamma=0$.

The approximation of the epipolar direction requires more attention than roughly guessing the rotation angles. Transforming the optical flow vectors to the surface of a unit sphere, the flow components perpendicular to the epipolar direction should cancel each other out when computing the sum of all the vectors, as shown in Fig. 3. The resultant 
vector points towards the epipole in this case. Thus, the approach taken in [1] is to obtain the initial values for $\theta$ and $\phi$ from the vector sum. However, as discussed in the next section, such an initial guess may lead to highly incorrect final results, especially in outdoor situations. This is the motivation behind applying semantic segmentation to improve the motion estimation.

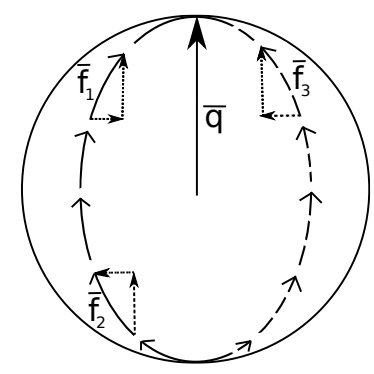

Fig. 3. Optical flow vectors along two epipolar lines on the opposing sides of the unit sphere, with the epipolar direction given by the vector $\vec{q}$. Three optical flow vectors $\overrightarrow{f_{1}}, \overrightarrow{f_{2}}$, and $\overrightarrow{f_{3}}$ are shown decomposed into their components in the epipolar direction and in the plane perpendicular to $\vec{q}$. The perpendicular component of $\vec{f}_{1}$ is canceled out by the corresponding component of either $\overrightarrow{f_{2}}$ or $\overrightarrow{f_{3}}$.

After solving the minimization problem, the motion parameters and optical flow vectors together are used to triangulate the 3D coordinates of the observed points. The reconstructed model can be refined by evaluating the optical flow again along the epipolar lines, but we skip this step for now because of our goal of nearly real-time operation. Further mathematical details of the entire reconstruction process can be found in [1].

\section{ImPRoving the ReCONSTRUCTION With Semantic SEgMENTATION}

A drawback of the reconstruction method described above is the decreased robustness in the presence of dynamic objects, uniform textures, and objects very close to or far away from the camera. All of these factors tend to be present in outdoor scenarios, but are also encountered in indoor settings, such as in the case of walls and floors with a uniform appearance. The modifications presented in the following are based on the acknowledgment that the presence of such objects may prevent the convergence of Eq. (1).

Compared to the work of [1], the following modifications are suggested: (i) the objects belonging to the problematic semantic classes are identified by a neural network trained on equirectangular images, (ii) an initial guess for the epipolar direction is obtained by setting the length of the optical flow vectors to zero in the 'unreliable' regions, (iii) the motion parameters are evaluated with Eq. (1) using the modified optical flow, and (iv) the 3D coordinates of the pixels in the reliable regions are obtained via triangulation.

\section{A. Problematic Semantic Classes}

For the reconstruction and motion estimation task, different classes of objects may be problematic for various reasons. Dynamic objects are especially challenging for the obvious reason that their optical flow vectors are composed both of their own motion and the movement of the camera. Without information from external sensors or motion models, the effect of the object motion cannot be removed from the optical flow vectors to obtain the flow component resulting only from the moving camera. Frequently encountered dynamic objects include humans, animals, and vehicles, but also semantic classes such as trees and the sky, which can be dynamic or static depending on the weather conditions.

Uniform or repeating textures notoriously cause issues when it comes to stereo matching techniques. They are also challenging in the context of the current work because we do not yet have a tool to identify the textures that are too uniform for a reliable optical flow estimation.

Additional difficulties arise from objects both close to and far away from the camera. Nearby objects appear distorted and may not be suitable for optical flow evaluation. On the other hand, the parallax of faraway objects cannot be observed and thus it makes no sense to attempt to triangulate their coordinates.

\section{B. Treatment of Unreliable Regions in Spherical Images}

As mentioned above, the flow vectors in the problematic regions are set to zero for a more robust evaluation of the direction of motion. This may seem like a simple solution, and it is necessary to consider both the initial approximation for the epipolar direction and the final optimization computed from Eq. (1) to understand why the idea works.
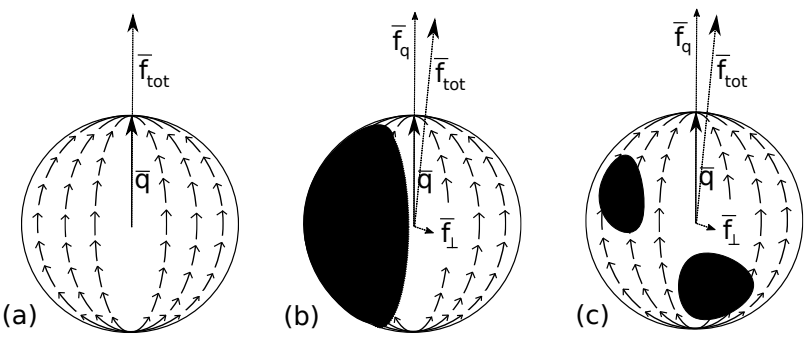

Fig. 4. (a) Optical flow vectors in the case of pure translational motion. The vector sum $\vec{f}_{\text {tot }}$ is approximately parallel to the epipolar direction $\vec{q}$ in the case where the all the optical flow vectors can be evaluated reliably. (b) Example case where nearly half of the optical flow vectors are set to zero in an unreliable region depicted by a black mask. The components of $\vec{f}_{\text {tot }}$ in the epipolar direction, $\overrightarrow{f_{q}}$, and in the perpendicular plane, $f_{\perp}$, are shown to demonstrate that the vector sum is very close to the epipolar direction. (c) Example case where small regions in different locations on the unit sphere are classified unreliable and set to zero, still resulting in $\vec{f}_{\text {tot }}$ rather close to the epipolar direction. 
In the case of a nearly ideal optical flow pattern, such as the one in Fig. 1, there is hardly any contribution from the problematic object classes mentioned above. The epipolar direction is approximated by the sum of all the flow vectors, which is expected to work because of the components canceling each other out. It is thus reasonable to ask what happens if some fraction of the flow vectors are set to zero, effectively dropping these vectors out of the sum being minimized in Eq. (1). In most cases, the result is still surprisingly close to the actual epipole. The explanation for this is illustrated in Fig. 4 , which shows the optical flow vectors on a unit sphere and masks covering parts of the sphere.

An ideal situation with no unreliable optical flow vectors is seen in Fig. 4 (a). The masked regions in Figs. 4 (b) and (c) correspond to the flow magnitudes being set to zero. In the case of Fig. 4 (b), nearly half of the image is masked, corresponding to the real-life situation where the sky takes up a large fraction of the image. Another possibility is illustrated in Fig. 4 (c), where unreliable regions are found randomly distributed around the unit sphere.

As more vectors are added to the vector sum in the cases of Figs. 4 (b) and (c), the component of the resultant vector in the epipolar direction gets increasingly longer. The component in the perpendicular direction, on the other hand, is composed of vector components in random directions. If some of these composing vectors are left out of the sum, they do not completely cancel each other out, but the perpendicular component of the resultant vector is still much shorter in length than the component in the epipolar direction. This indicates that the resultant vector approximately points in the correct direction, at least unless a highly large fraction of the optical flow vectors is deemed unreliable.

In the final optimization phase, a large number of optical flow vectors with zero magnitude should not interfere with the final result. Due to the uncertainty of the number of unmodified flow vectors needed for an accurate initial guess and final result, it is necessary to check the quality of the solution using some suitable condition, such as checking if the average angle between the rotation-corrected optical flow vectors and the epipolar lines is below an acceptable maximum value.

It should be noted that some of the unreliable regions may contain a large number of nearly zero-length flow vectors even if they are not specifically set to zero, such as in the case of a clear sky with no moving objects. In such cases, the main benefit of the semantic classification is keeping us from spending computational resources on attempting triangulation in these regions.

\section{Semantic Segmentation}

Image segmentation is a machine vision task where we do labeling of a specific object or region within the frame or image. Here, we have developed a Deeplab V3+ model to predict different classes such as sky, asphalt, etc.

\section{A. Dataset Preprocessing}

In this dataset compilation, we collected around 12400 frames of different scenarios which have semantic classifications like sky, trees, gravel, asphalt, tiles, and grass. The images were captured using the Insta360 ONE X Camera with a fisheye lens. The annotation was done manually using Labelme: Image Polygonal Annotation with Python [11] and 649 images chosen randomly for annotation. The pixels other than those segmented in the represented classes were considered as background. The coordinates of the annotated targets in each image were recorded in an extensible markup JSON file with their classified labels.

\section{B. Semantic Segmentation using Deeplab}

The aim of semantic segmentation is to classify each pixel of an image with a respective classification of a represented object. This is referred to as dense prediction since we are predicting each and every pixel of the image.

The construction of neural network architectures for this task simply consists of defining the number of convolutional layers and predicting the output segmented images. The preprocessed dataset was used as the training and test data for the DeeplabV3+ semantic segmentation network $(75 \%$ as training data, $25 \%$ as test data). The fine-tuned ResNet-101 was used to construct the Deeplab V3+ network, a semantic segmentation model designed to achieve the segmentation of the specific target. ResNet-101 [12] is well suitable for ground-level object segmentation due to its residual learning framework. Another reason for choosing ResNet-101 is its suitability for a small-scale dataset. The processing of full resolution images throughout the network is computationally expensive and it makes the process extremely slow, so we decided to downsample the images with respect to their spatial resolution to $512 \times 512$.

Deeplab V3+ can control the feature extraction by an encoder and improve the segmentation accuracy of the specific target boundaries [13]. At the encoder stage, the connection layer and subsequent layers in ResNet-101 were removed to complete the feature extraction. In order to measure the segmentation accuracy of the Deeplab V3+ model constructed for this project, we used the trained model to complete the target segmentation of the test set, selected mIoU (mean Intersection over Union) and accuracy of the target segmentation. The results suggest that the accuracy of the segmentation of the target reached a mIoU of $78.41 \%$ for the sky and $72.48 \%$ for trees, which indicates that the Deeplab v3+ model based on ResNet-101 could achieve highprecision segmentation of these targets. The other classes are not predicted as reliably because of the small scale of the dataset that was focused on few classifications. The segmentation results are shown in Fig. 5 (b). The proposed model should be able to predict the sky and trees. Our future 

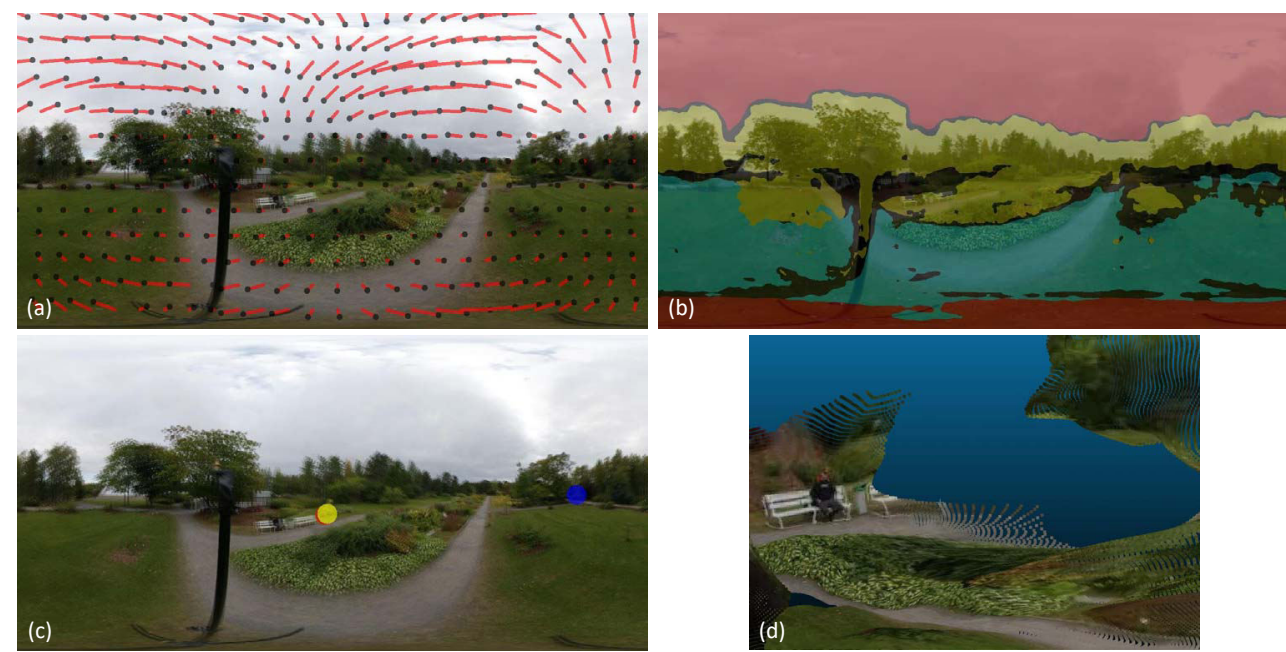

Fig. 5. Example 1: Garden scene. (a) Optical flow pattern between two spherical images. (b) Results of the segmentation by the Deeplab network. The segmentation will be improved in our future work after collecting more spherical image data. The current results were applied to set the optical flow vectors to zero in the sky region. (c) The blue dot represents the initial estimation for the epipole in the case where none of the optical flow vectors are modified. The yellow dot is the initial guess in the case where the vectors in the masked region are set to zero, and the red dot shows the final estimate for the epipolar direction after the optimization. Note that the initial guess and the final estimate are very close to each other. (d) Detail from the 3D reconstruction of the garden.

work will include the collection of more spherical data to improve the capability of the neural network to recognize highly distorted objects.

\section{EXPERIMENTS}

The reconstruction technique was tested with spherical images from Insta360 ONE X. The equirectangular image projections were obtained from the Insta360 software.

Two example cases of semantic 3D reconstruction from equirectangular images are presented below. The optical flow was evaluated with the Volumetric Correspondence Network ([14]), which is suitable for distorted images. The neural network described above was used to identify the sky in Example 1. Manual segmentation was applied in Example 2 because it requires the identification of a distorted car and regions covered in asphalt, for which the neural network does not yet produce satisfactory results.

The optical flow patterns may be compared to those in Fig. 1 , which contains no dynamic objects (note that the person in the image is standing still), uniform regions, or objects at very short or long distances from the camera. In this sense, the patterns seen in Fig. 1 are close to ideal for the purpose of the reconstruction task. Also, it is easy to estimate the location of the epipole by eye just by finding the point that seems to be the origin of the flow.

\section{A. Example 1: Garden with Moving Clouds}

The first example features two images from a garden on a windy day with moving clouds in the sky. Comparing the optical flow patterns of Fig. 5 (a) to the ideal case of Fig. 1 , it appears that the texture of the grass and gravel at the ground level is varied enough for a reliable estimation of the flow vectors. In the sky, the clouds have clearly moved between the two frames: the average flow magnitude of the clouds is far from zero and is actually higher than the average magnitude of the ground-level flow vectors, which result from the camera motion alone. The flow vectors are set to zero in the sky region identified by the neural network, the results from which are illustrated in Fig. 5 (b).

The initial epipolar estimates in Fig. 5 (c) demonstrate that the motion of the clouds causes the initial guess to drift far away from its expected location. Setting the optical flow of the clouds to zero, the initial value greatly improves and is quite close to the location of the epipole inferred from the flow patterns seen on the ground. The detail from the resulting 3D model in Fig. 5 (d) shows that the reconstruction has been fairly successful even at ground level.

The most important conclusions from this example are (i) that setting the optical flow in the sky to zero may lead to a considerable improvement of the evaluated epipolar direction, and (ii) that 3D reconstruction at the ground level is possible for at least some grass and gravel textures, which supports the idea of further research into the recognition of textures suitable for reconstruction based on optical flow vectors. 

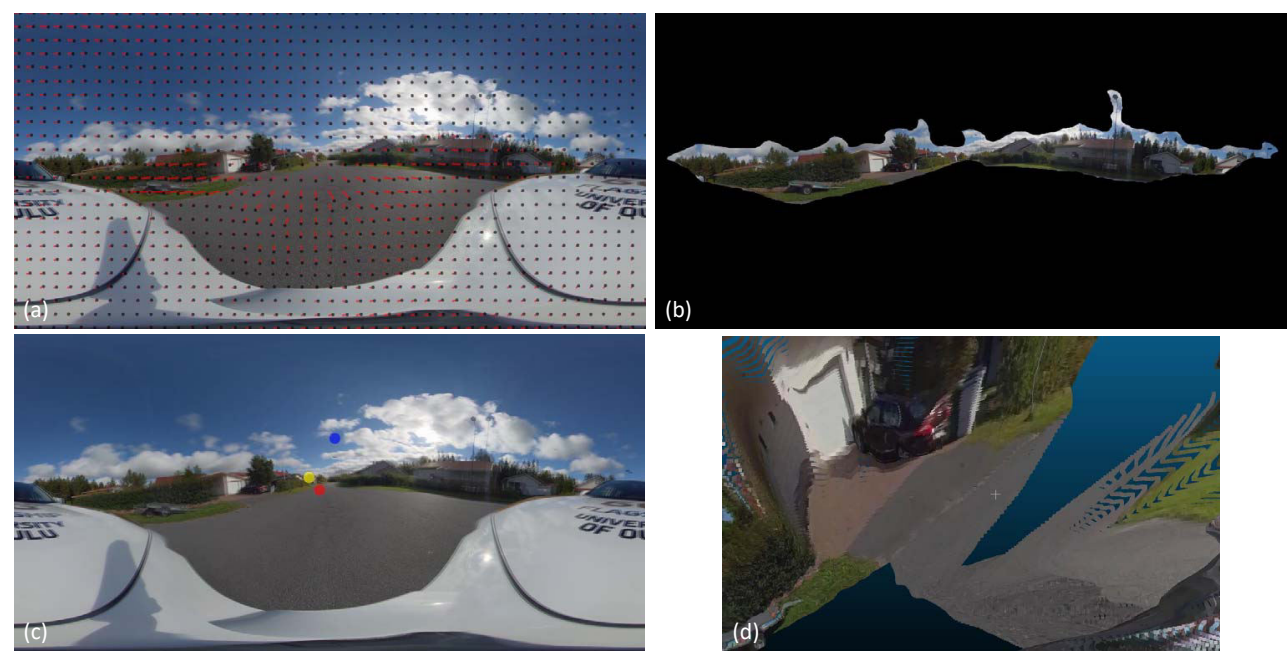

Fig. 6. Example 2: Driving scene. (a) Optical flow pattern between two spherical images. (b) The black mask covers the manually segmented regions containing sky, asphalt, and the visible parts of the car that the camera is attached to. (c) The blue dot represents the initial estimation for the epipole in the case where none of the optical flow vectors are modified. The yellow dot is the initial guess in the case where the vectors in the masked region are set to zero, and the red dot shows the final estimate for the epipolar direction after the optimization. (d) Detail from the 3D reconstruction of the asphalt road together with a building and a car at the side of the road.

\section{B. Example 2: Driving Scene with Large Problematic Re- gions}

In this challenging example, the camera is attached to the front of a moving car. A large fraction of the image is covered by either the sky or the car itself, and the optical flow in these regions is approximately equal to zero, as seen in Fig. 6 (a). The asphalt-covered road also takes up a large area in the image, and it is uncertain whether the flow vectors of the road can be used for reconstruction.

The segmentation of the areas of interest was done manually, and these regions correspond to the masked areas in Fig. 6 (b). Again, the optical flow is set to zero in the masked regions. Looking at the estimated epipolar directions in Fig. 6 (c), the initial guess for the epipolar direction improves judging by the optical flow patterns of the observed buildings and the expected direction of motion of the car.

After evaluating the motion parameters, the reconstruction is done not only for the unmasked regions but also for the road. This is motivated by the goal to inspect what kind of ground-level textures are suitable for reconstruction with the optical flow method. The detail from the 3D model shown in Fig. 6 (d) indicates that only a small fraction of the road has been accurately triangulated. The texture is too uniform for this purpose farther away from the camera, and the motion of the car is too fast to track the motion of the part of the road closest to the car because it moves out of view between the two frames. Like the previous example, this encourages further research into classification of surfaces based on their applicability for this reconstruction method.

\section{CONCLUSION}

The method described in the paper makes it possible to reconstruct 3D structures from spherical images based on optical flow vectors in typical outdoor scenarios and also indoor scenarios with uniform textures. The basic idea is to use semantic segmentation to find the regions in which it is likely to find unreliable optical flow vectors, and the magnitude of these vectors is set to zero for a more robust evaluation of the camera motion.

The further development of the presented method depends on whether the focus is on geometric accuracy or real-time applications. Because of the demand for real-time mapping in mobile robotics, one viable option for a robot is to utilize the method to construct a geometrically rough map with accurate semantic classifications. The robot can then use this map to find the relevant objects related to its task together with their approximate locations and physical extent. If the robot carries an additional sensor with a higher metric accuracy but a narrowe field of view, such as an RGB-D camera, it can then focus the more accurate observations on the objects of interest. This can lead to a more cost-efficient mapping process, as fewer observations are required and memory usage can be reduced by storing the less important regions of the map at a lower resolution.

\section{ACKNOWLEDGMENT}

The authors would like to thank the NUVE-LAB - Developing Autonomous and Environmentally Sustainable Utility Vehicles project for the financial support. 


\section{REFERENCES}

[1] S. Pathak, A. Moro, A. Yamashita, and H. Asama, "Dense 3D reconstruction from two spherical images via optical flow-based equirectangular epipolar rectification," in IEEE International Conference on Imaging Systems and Techniques (IST), 2016, pp. 140-145.

[2] S. Pathak, A. Moro, H. Fujii, A. Atsushi, and H. Asama, "Distortionrobust spherical camera motion estimation via dense optical flow," in 25th IEEE International Conference on Image Processing (ICIP), 2018, pp. 3358-3362.

[3] T. da Silveira and C. Jung, "Dense 3D Scene Reconstruction from Multiple Spherical Images for 3-DoF+ VR Applications," in IEEE Conference on Virtual Reality and 3D User Interfaces (VR), 2019, pp. 9-18.

[4] S. Im, H. Ha, F. Rameau, H. Jeon, G. Choe, and I. Kweon, "Allaround depth from small motion with a spherical panoramic camera," in European Conference on Computer Vision, 2016, pp. 156-172.

[5] J. Huang, Z. Chen, D. Ceylan, and H. Jin, "6-DOF VR videos with a single 360-camera," in IEEE Virtual Reality (VR), 2017, pp. 37-44.

[6] H. Jang, D. Jeon, H. Ha, and M. Kim, "Fast Omnidirectional Depth Densification," in International Symposium on Visual Computing, 2019, pp. 683-694.

[7] I. Uygur, R. Miyagusuku, S. Pathak, A. Moro, A. Yamashita, and H. Asama, "Robust and Efficient Indoor Localization Using Sparse Semantic Information from a Spherical Camera," Sensors, vol. 20, no. 15, p. 4128, 2020.

[8] G. Pintore, Giovanni, F. Ganovelli, R. Pintus, R. Scopigno, and E Gobbetti, "3D floor plan recovery from overlapping spherical images," Computational Visual Media, vol. 4, no. 4, pp. 367-383, 2018.

[9] N. Zioulis, A. Karakottas, D. Zarpalas, and P. Daras, "Omnidepth: Dense depth estimation for indoors spherical panoramas," in Proceedings of the European Conference on Computer Vision (ECCV), 2018, pp. 448-465.

[10] M. Lourakis, "A brief description of the Levenberg-Marquardt algorithm implemented by levmar," Foundation of Research and Technology, vol. 4, no. 1, pp. 1-6, 2005.

[11] K. Wada. Labelme: Image Polygonal Annotation with Python. (2016). [Online]. Available: https://github.com/wkentaro/labelme

[12] K. He, X. Zhang, S. Ren, and J. Sun, ”Deep Residual Learning for Image Recognition," in IEEE Conference on Computer Vision and Pattern Recognition (CVPR), 2016, pp. 770-778.

[13] L. Chen, G. Papandreou, I. Kokkinos, K. Murphy, and A. Yuille, "Deeplab: Semantic image segmentation with deep convolutional nets, atrous convolution, and fully connected crfs," IEEE transactions on pattern analysis and machine intelligence, vol. 40, no. 4, pp. 834-848, 2017.

[14] G. Yang and D. Ramanan, "Volumetric Correspondence Networks for Optical Flow," NeurIPS, vol. 5, p. 12, 2019. 